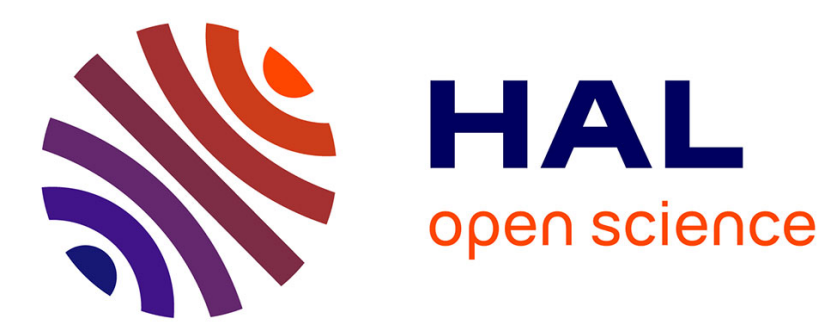

\title{
Relevés des colonnes de la grande salle hypostyle de Karnak (note d'information)
}

\author{
Emmanuel Laroze, Bertrand Chazaly
}

\section{To cite this version:}

Emmanuel Laroze, Bertrand Chazaly. Relevés des colonnes de la grande salle hypostyle de Karnak (note d'information). Comptes-rendus des séances de l'Académie des inscriptions et belles-lettres, 2009, 153 (2), pp.669-685. 10.3406/crai.2009.92528 . hal-02590689

\section{HAL Id: hal-02590689 \\ https://hal.science/hal-02590689}

Submitted on 15 May 2020

HAL is a multi-disciplinary open access archive for the deposit and dissemination of scientific research documents, whether they are published or not. The documents may come from teaching and research institutions in France or abroad, or from public or private research centers.
L'archive ouverte pluridisciplinaire HAL, est destinée au dépôt et à la diffusion de documents scientifiques de niveau recherche, publiés ou non, émanant des établissements d'enseignement et de recherche français ou étrangers, des laboratoires publics ou privés. 


\title{
NOTE D'INFORMATION
}

\author{
RELEVÉS DES COLONNES \\ DE LA GRANDE SALLE HYPOSTYLE DE KARNAK, \\ PAR MM. EMMANUEL LAROZE ET BERTRAND CHAZALY
}

\section{Problématique}

Nous connaissons une période de développements technologiques remarquables, qui renouvellent l'ensemble des méthodes de recherche dans des disciplines anciennes, parfois attachées à leurs outils traditionnels. C'est le cas de l'archéologie, qui oriente aujourd'hui les toutes nouvelles technologies de pointe vers l'exploration du passé, et offre des terrains d'application particulièrement favorables à leur développement.

Ces avancées technologiques ont en retour un fort impact sur les résultats : en s'appuyant sur un éventail d'instruments plus larges et innovants, notre capacité à interpréter les contextes archéologiques progresse. L'informatique, bien sûr, est la première grande avancée à avoir pénétré le domaine de l'archéologie. Le développement d'outils informatiques adaptés directement à l'archéologie ou dérivés d'autres applications ont permis des évolutions marquantes, en matière d'archivage, de traitement des données, de méthodologie, et de communication. Le prestige des sites, mais surtout le défi que constituent certains contextes, font de l'archéologie un domaine de prédilection pour l'expérimentation d'outils inédits et l'application en situations réelles. Le temple de Karnak, par la richesse de son histoire, la grandeur de ses vestiges et la complexité de ses structures, n'est-il pas un cadre idéalement problématique pour la mise en pratique des technologies les plus performantes?

Avec le Centre franco-égyptien d'Étude des Temples de Karnak, le site de Karnak ${ }^{1}$ est devenu le terrain d'entreprises ambitieuses sur les thèmes «Archéologie et Technologie »: "L'assemblage des

1. www.cfeetk.cnrs.fr 
talatat à l'aide de l'ordinateur $»^{2}$ ou $«$ Les grandes étapes d'évolution du temple d'Amon-Rê dessinées à l'ordinateur » ${ }^{3}$, ont eu dans les années 1980 un grand retentissement. Pour conduire ces projets, le Centre franco-égyptien a travaillé en collaboration avec la direction des Études et Recherches d'Électricité de France ${ }^{4}$, et a bénéficié du prix «Sphère du Mécénat » attribué par le jury de l'Institut de France et la Fondation Fiat-France. L'Académie des Inscriptions et Belles-Lettres, par l'intermédiaire de son secrétaire perpétuel, Jean Leclant, a ainsi toujours encouragé quand elle le pouvait des programmes novateurs en archéologie ${ }^{5}$.

Le projet du relevé des colonnes de la grande salle hypostyle de Karnak, n'aurait pas pu voir le jour sans le soutien renouvelé de l'Institut de France, qui a décerné en 2005 le prix de la Fondation Simone et Cino del Duca au Centre franco-égyptien.

Pour les milliers de visiteurs quotidiens qui découvrent le grand temple dynastique d'Amon-Rê à Karnak, la salle hypostyle, est sans nul doute la partie la plus spectaculaire du monument. Ici, l'architecture est un véritable support aux textes ; pas un mur, pas une colonne n'est dépourvue de décors gravés représentant des scènes rituelles ou sacrées. La densité de l'épigraphie qui a parfois préservée ses couleurs, est saisissante.

Pourtant, aussi célèbre soit elle, la grande salle demeure mal documentée : jusqu'à récemment, il n'existait aucun relevé photographique exhaustif des décors, nécessaire à une analyse détaillée. Une partie de cette lacune a été comblée ces dernières années par une importante campagne de relevé photographique en noir et blanc. Cette étude conduite par le Centre franco-égyptien, a concerné

2. R. Vergnieux, Recherches sur les monuments thébains d'Amenhotep IV à l'aide des outils informatiques, Cahiers de la Société d'Égyptologie vol. 4, Genève, 1999 ; S. Billet, M. Gondran, R. Vergnieux, «Le puzzle d'Akhenaton reconstitué à Karnak », Dossiers de l'Archéologie n ${ }^{\circ} 153$ octobre 1990, p. 58-63 ; R. Vergnieux, «Premiers exemples de résultats obtenus à l'aide du fichier informatisé sur les talatat et vocabulaire de recherche ", Wissenschaftliche Zeitschrift der Humboldt-Universität zu Berlin 37/3, 1988, p. 283-287.

3. J.-Cl. Golvin, «Quelques travaux récents du Centre franco-égyptien de Karnak, 1985-1988 », Comptes Rendus de l'Académie des Inscriptions et Belles-Lettres, 1988, Volume 132, Numéro 3, p. 575-599; H. Boccon-Gibod et J.-Cl. Golvin, « Le grand temple d'Amon-Rê à Karnak, reconstruit par l'ordinateur », Dossiers de l'Archéologie n¹53, octobre 1990, p. 8-19.

4. Ce travail a abouti à un ouvrage collectif : M. Albouy, H. Boccon-Gibod, J.-C. Golvin, J.-Cl. Goyon, P. Martinez, Karnak, le temple d'Amon restitué par l'ordinateur, Solar, 1991. Concernant le mécénat technologique voir M. Albouy, « Le mécénat technologique et scientifique au service de l'archéologie », Dossiers de l'Archéologie n ${ }^{\circ} 153$, octobre 1990, p. 2-7.

5. Voir les observations de Jean Leclant dans Comptes rendus de l'Académie des Inscriptions et Belles-Lettres, 1988, volume 132, numéro 3, p.598-599. 
les parois périphériques de la salle et a été publiée sous la forme d'un DVD'.

Cependant, pour compléter cette documentation, il restait à traiter les colonnes. La tâche demeurait délicate à cause de la géométrie du support : l'aspect non planaire lié au galbe des colonnes pose des problèmes de projection et de représentation auxquels les outils traditionnels - relevés manuels sur plastiques, photographie - ne peuvent pas répondre ${ }^{7}$. Ainsi, l'opération se résume à une procédure bien connue mais qui reste complexe : produire une image planaire (2D) à partir d'une représentation appliquée sur un volume (3D). La photogrammétrie combinée au scannage $3 \mathrm{D}$ a permis de résoudre ces difficultés.

Ce programme a donné l'occasion de compléter une documentation encore partielle, mais également d'explorer, à travers un contexte particulièrement complexe, des techniques innovantes de relevé appliquées à l'archéologie. Plusieurs compétences issues de l'ingénierie comme des sciences humaines, se sont croisées sur ce programme, et l'aventure a été collective : trois institutions publiques sont intervenues, une égyptienne et deux autres françaises - le Conseil Supérieur des Antiquités d'Égypte (CSA), le CNRS, l'École nationale des Sciences Géographiques (ENSG-IGN) ${ }^{8}$, ainsi qu'une entreprise privée spécialisée dans la numérisation 3D par la sergrammétrie : Atm3 $\mathrm{D}^{9}$.

\section{Présentation de la salle}

La salle hypostyle de Karnak succède à la grande cour et forme une sorte d'anti-chambre avant l'entrée dans le temple proprement dit : l'Ipet-Sout. Selon les textes des architraves, la salle est considérée comme un " temple de millions d'années »-c'est-à-dire un lieu de célébration du culte monarchique associé au culte d'Amon. Le décor polychrome intérieur évoque les cérémonies accomplies en ce lieu, comme la fête de la barque sacrée et le rituel divin journalier,

6. A. Arnaudiès, A. Chéné, Les parois de la salle hypostyle de Karnak, DVD publié aux éditions Cybèle, Paris, 2003. Le DVD a été présenté par le Professeur Nicolas Grimal devant l'Académie des Inscriptions et Belles-Lettres le 27 juin 2003.

7. Le problème s'est posé pour les colonnes du temple d'Esna par exemple. S. Sauneron, Quatre campagnes à Esna, ESNA 1, IFAO, Le Caire, 1959. p. 166-167.

8. www.ensg.ign.fr.

9. www.atm3d.com. 
tandis que le décor extérieur illustre les victoires de Séthi I ${ }^{\mathrm{er}}$ au Nord, et de Ramsès II au Sud.

Il semble que Séthi I ${ }^{\text {er }}(-1291$ à -1278$)$ soit à l'origine de la construction de la salle ${ }^{10}$. Ce souverain de la XIX ${ }^{\mathrm{e}}$ dynastie, aurait ordonné l'érection des 134 colonnes entre les $\mathrm{II}^{\mathrm{e}}$ et $\mathrm{III}^{\mathrm{e}}$ pylônes de ses prédécesseurs, Horemheb (-1323 à - 1295) et Amenhotep III $(-1391$ à - 1353), transformant ainsi une aire d'environ un demihectare en un vaste espace couvert. Les douze grandes colonnes papyriformes à chapiteau ouvert encadrent l'allée centrale, tandis que les 122 autres supportent les couvertures des nefs latérales. De grands claustras, installés à l'articulation des deux niveaux de toiture, assurent l'éclairage de la voie centrale ; le reste de la salle, percé de petites ouvertures zénithales restait plongé dans la pénombre. Séthi I ${ }^{\text {er }}$ entreprit par ailleurs la décoration de colonnes mais ne put malheureusement pas achever son projet : seule la moitié nord de la salle et les claustras de la nef centrale portent ses cartouches. Ses successeurs, Ramsès II $(-1278$ à -1212$)$ puis Ramsès IV (- 1151 à - 1145) parachevèrent l'ornementation de la salle.

\section{L'histoire du projet}

Le relevé des colonnes de la salle hypostyle n'est pas une question récente. Il y a une quinzaine d'années déjà, le photographe Antoine Chéné, ingénieur d'étude au CNRS qui dirigeait alors le service de photographie du Cfeetk, avait proposé une première solution technique au problème. Il s'agissait d'adapter un ingénieux procédé de photographie «en développé », mis au point et breveté par lui-même, pour les décors de vases : la périphotographie ${ }^{11}$.

Le principe consiste à faire tourner le vase devant un appareil photographique fixe, l'obturateur ouvert ; le film se déroule alors simultanément au mouvement de rotation du vase. Grâce à ce système de prise de vue, on obtient une représentation déroulée du décor. Pour l'appliquer à des supports cylindriques de plus grandes dimensions tels que des colonnes ou des bornes milliaires, Antoine Chéné a développé une variante du système :

10. P. Brand, The Monuments of Seti I : Epigraphic : Historical and Art-Historical Analysis, Brill, Leiden, 2000, p. 212

11. A. Chéné, P. Foliot, G. Réveillac, La pratique de la photographie en archéologie, Edisud, Aix-en-Provence, 1999, p. 119-122. 
le périgyrophot ${ }^{12}$. L'objet ne pouvant pas subir de rotation, c'est l'appareil photographique lui-même, monté sur un rail circulaire, qui tourne autour de la colonne. Les prises de vues sont faites dans l'obscurité et l'homogénéité de l'éclairage est assurée par un projecteur tournant en même temps que l'appareil photographique.

C'est dans le cadre d'un projet documentaire total des décors de la salle hypostyle qu'a été envisagé l'usage du périgyrophot, seul moyen technique de l'époque, qui s'est pourtant heurté à deux problèmes majeurs : les dimensions extrêmes des colonnes et leur nombre. Leurs diamètres variant de 2 à $3,5 \mathrm{~m}$ et leurs hauteurs de 13 et $20 \mathrm{~m}$, une longue mise au point aurait été nécessaire pour adapter le système au gigantisme de la salle. La gestion des échafaudages en particulier, était délicate compte tenu des hauteurs à atteindre et chose encore plus décourageante, ils devaient être démontés et réassemblés autant de fois qu'il y a de colonnes. Par ailleurs, le système ne pouvait fonctionner que pendant la nuit, et devait encombrer durablement la salle d'échafaudages ; l'installation était par conséquent difficile à concilier avec la vocation touristique du lieu. Face à la lourdeur des dispositifs à mettre en place, le projet de documentation s'est tourné dans un premier temps sur la couverture photographique des parois plane de la salle, les murs périphériques.

Plus récemment, les professeurs Jean Revez et Claude Parisel, partenaires du groupe de recherche en conception assistée par ordinateur (GRCAO) de l'Université de Montréal ${ }^{13}$, se sont de nouveau intéressés à la problématique du relevé des colonnes de la salle. Dans le cadre de ce programme, Élise Meyer a proposé une solution technique mêlant de la topographie avec des clichés photographiques pour représenter un décor appliqué sur des surfaces non planes ${ }^{14}$.

12. A. Chéné, «Une nouvelle méthode pour photographier les inscriptions sur support cylindrique : le périgyrophot », Bulletin de l'École Antique de Nîmes 20, 1990, p. 113-118.

13. Le site de Karnak est pour le groupe de recherche un inépuisable champ d'étude, voir la bibliographie complète sur le site : http://www.grcao.umontreal.ca ; voir également É. Meyer, C. Parisel, P. Grussenmeyer, J. Revez, T. Tifadi, «A computerized solution for the epigraphic survey in Egyptian Temples », Journal of Archaeological Science 33.11, nov. 2006, p. 1605-1616.

14. É. Meyer, Modélisation photogrammétrique de l'épigraphie des colonnes de la salle hypostyle du temple de Karnak, Mémoire de soutenance de stage du DEA « Modélisation et simulation des espaces bâtis », École d'architecture de Nancy, octobre 2003. (http://www.crai.archi. fr/media/pdf/E_Meyer_memoireDEA0203.pdf) ; É. Meyer, « La photogrammétrie pour le relevé épigraphique des colonnes de la salle hypostyle du temple de Karnak », Revue XYZ 102, 2005, p. 31-34. (http://halshs.archives-ouvertes.fr/halshs-00262171/en) 
Depuis, les outils technologiques appliqués aux relevés se sont perfectionnés, et grâce au développement de la technique de la lasergrammétrie, l'usage du scannage 3D s'est démocratisé. Autrefois réservé à l'activité industrielle, cet instrument est maintenant régulièrement employé en archéologie. En parallèle, l'essor de la photographie numérique associée à l'augmentation de la puissance de calcul des ordinateurs ont ouvert de nouvelles perspectives en matière d'acquisition d'images, et la globalisation des instruments numériques a favorisé les échanges entre différentes disciplines. En particulier, le rapprochement entre la photographie et la topographie, jadis deux domaines aux activités bien distinctes, a permis des développements à la fois techniques et méthodologiques tout à fait nouveaux ${ }^{15}$. Par exemple, la densité des nuages de points topographiques issus des scanners 3D est désormais telle qu'on pourrait la qualifier de photographie 3D. Réciproquement, le simple appareil photographique est aujourd'hui devenu un capteur extrêmement puissant avec lequel, par des procédés de photogrammétrie, il est possible de produire des modèles numériques tridimensionnels très denses. Ce sont sur les bases de ces nouvelles technologies, totalement numériques, que notre projet s'est développé.

Le temple d'Opet, objet d'une étude architecturale, a été le premier terrain d'application et d'expérimentation de ces nouveaux instruments de relevés, sur le site de Karnak ${ }^{16}$. En moins de deux semaines toutes les données nécessaires à l'élaboration d'un modèle numérique complet du temple ont été enregistrées, permettant de produire un nombre illimité de sections sur le monument. C'est dans la continuité de ces résultats très encourageants que les premiers tests ont commencé à l'automne 2005 dans la salle hypostyle.

Cette première intervention a porté sur la colonne 2 n, située au Nord-Est de la nef centrale, qui permettait d'appréhender concrètement les difficultés liées aux conditions de terrain : proximité du pylône est, flux touristique important, dimensions et état de la colonne, nombreuses traces de couleur.

15. À propos des développés orthotphotographiques voir : G.E. Karras, P. Patias, E. Petsa K. Ketipis, "Raster projection and development of curved surfaces », International Archives of Photogrammetry \& Remote Sensing 32 (5C1B), 1997, p. 179-185 ; B. Chazaly, « Cylindrical orthophotography - Complete coverage of inside/outside cylindrical surfaces such as castle towers ", Proceedings XVIII ${ }^{\text {th }}$ international symposium of CIPA, Potsdam. Article en ligne : http:// cipa.icomos.org/POSTDAM.html.

16. B. Chazaly et E. Laroze, « Le relevé par scannage 3D du temple d'Opet à Karnak », Revue $X Y Z 102,2005$, p. 25-30. Les premiers tests de scannage eurent lieu entre le 22 et le 31 octobre 2005. (www.atm3d.com/v5/pdf/opet.pdf) 
Quatre positions de scanner ont été nécessaires pour couvrir intégralement la colonne, permettant l'enregistrement de plus de huit millions de points à sa surface, à une précision globale de quatre millimètres. Seize séries de cinq prises de vues photographiques en haute résolution ont complété la numérisation, offrant huit points de vue détaillés pris à deux moments différents de la journée - le matin et en fin d'après midi - afin de réduire l'incidence des ombres sur le monument.

Cet essai a fourni des informations importantes sur les conditions à respecter pour assurer un enregistrement correct de la géométrie d'une colonne et de son épigraphie. En outre, il a permis d'élaborer une procédure d'intervention visant, compte tenu du nombre de colonnes, à optimiser au maximum l'opération : la méthodologie développée pour scanner une colonne, n'est pas la même lorsqu'il s'agit d'en numériser 134.

Le couplage de la technique du scannage 3D à une couverture photographique, s'est avéré être une solution efficace pour produire une représentation déroulée des décors. Techniquement, la procédure élaborée nécessite l'association de deux types de données : un modèle numérique 3D de la salle - c'est-à-dire une représentation de la géométrie du support - et une couverture complète d'images photogrammétriques des décors. La mise en place d'un réseau topographique très précis est également requis pour permettre de géoréférencer et donc d'unifier l'ensemble de ces données. Les capteurs et les techniques d'acquisition n'étant pas les mêmes, l'opération s'est déroulée en deux étapes : une mission de numérisation 3D à l'automne 2007 puis une mission de prises de vue photogrammétriques au printemps $2008{ }^{17}$.

\section{La numérisation $3 D^{18}$}

La première mission a été consacrée au relevé complet de la salle par numérisation 3D (fig. 1). Cette science ou technique en plein essor, appelée également lasergrammétrie ${ }^{19}$, met en œuvre des

17. G. Véron, « 134 colonnes à la une », Le journal du CNRS 231, avril 2009, p. 28-30 ; Anonyme, «L'Ensg au temple du soleil », Ign Magazine 49, 2008, p. 16-17.

18. La mission s'est déroulée entre le $1^{\text {er }}$ et le 30 novembre 2007. L'équipe était composée de, Yvan Robin (Atm3d), Marion Saillant (Atm3d), Antoine Marmousez (Cfeetk/Mae) et Louis Elia (Cfeetk/Csa).

19. B. Chazaly, « La lasergrammétrie appliquée à l'auscultation des ouvrages d'art », Revue $X Y Z 107,2006$, p. 18-21. 


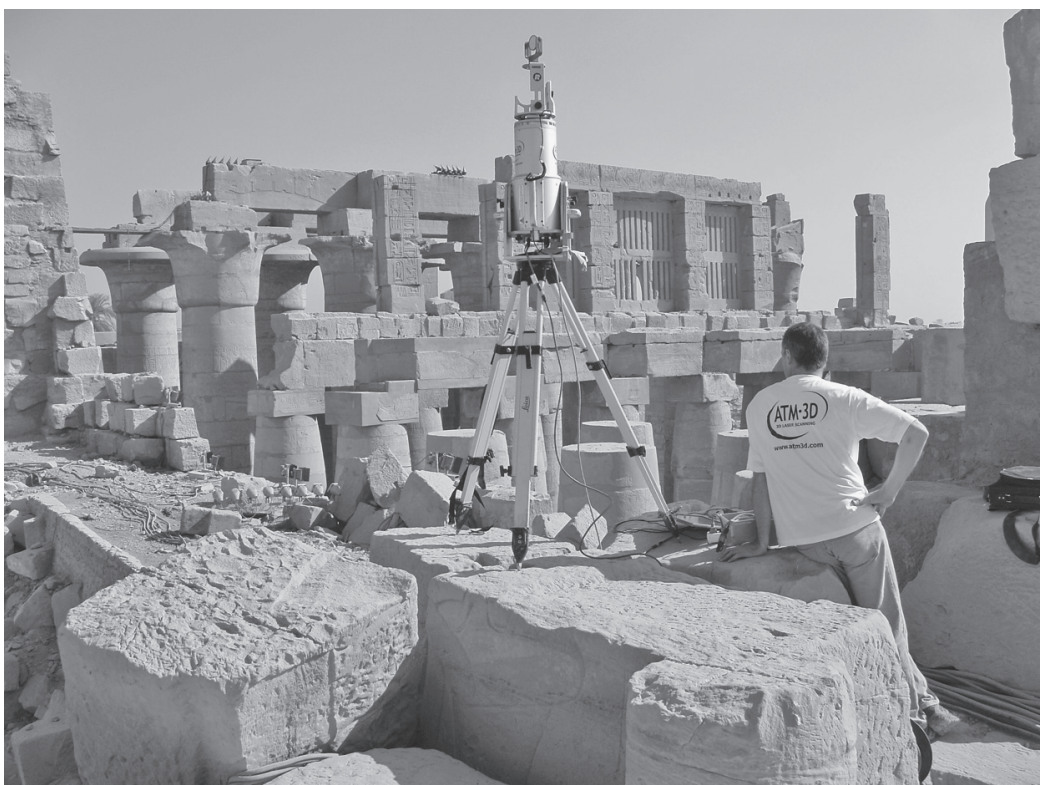

FIG. 1. - Le scanner en action dans l'angle sud-ouest de la salle hypostyle (E. Laroze).

scanners laser 3D (mesure laser haute densité, sans contact et longue portée). Les technologies de numérisation 3D ont trente ans : l'Institut de Technologie de l'Information du Conseil National de Recherche du Canada fut l'un des premiers laboratoires à mettre au point un scanner 3D, en $1978^{20}$. Mais c'est au début des années 1990 que les premiers appareils sont commercialisés. En France, deux réalisations spectaculaires témoignent des débuts du levé haute densité : la numérisation de la Grotte Cosquer (automne 1994) ${ }^{21}$ et celle du colosse d'Alexandrie (1997) ${ }^{22}$. Ces exemples marquants n'ont pourtant utilisé « que quelques » centaines de milliers de points.

20. M. Rioux, L. Cournoyer, « Travaux préliminaires à la mise au point d'un capteur de vision tridimensionnelle », NRC/ERB-944, Juin 1982, 16 pages, NRC 20189.

21. Voir à ce sujet : M. Paramythioti, «Grotte Cosquer : levé d'intérieur par le capteur Soisic », Revue XYZ 66, 1996, p. 24-27 ; M. Blaustein, "Grotte Cosquer : restitution photogrammétrique », Revue XYZ 68, 1996, p. 17-20 ; B. Brillault, F. Guisnel, G. Thibault, La grotte Cosquer au plus près du réel, G. Edf-Der, Clamart, 1996 ; Une visite virtuelle de la grotte est possible sur le site du Service Régional de l'Archéologie de la Direction Régionale des Affaires Culturelles ProvenceAlpes-Côte d'Azur : http://www.paca.culture.gouv.fr/dossiers/cosquer/html/intro.html.

22. Voir le bulletin d'information d'Edf : Aster Echos n' 28 novembre 1998 ; M. Paramythioti, « Le colosse d'Alexandrie », Revue XYZ 76, 1998. 
Aujourd'hui, un scanner 3D lève plusieurs millions de points en trois dimensions en quelques minutes, avec une précision millimétrique. Les capteurs dits longue portée peuvent numériser des objets distants de plusieurs centaines de mètres. Le principe est d'enregistrer en 3D tout ce qui est visible dans le champ de vision de l'appareil, généralement sur $360^{\circ}$.

Comme un scanner à plat, un scanner 3D enregistre une image à partir d'une série de mesures en lignes et en colonnes. À chaque élément de l'image, chaque " pixel », est associé l'information de la distance entre l'appareil et l'objet numérisé. La connaissance de la position et de l'orientation du scanner, qui s'obtient généralement par la numérisation préalable de repères connus en coordonnées, permet de convertir ces pixels en points 3D. L'information enregistrée par l'appareil est donc une image numérique. Le pas entre chaque ligne correspond à une résolution angulaire horizontale, entre chaque colonne à une résolution angulaire verticale. Ce pas est réglé par l'opérateur et fixe la densité des points $3 \mathrm{D}$, en fonction de la distance du scanner à l'objet.

Le paramètre de base étant la résolution angulaire de l'appareil, la densité des points mesurés à la surface de l'objet à numériser varie beaucoup selon la distance et l'orientation de chaque élément de surface de l'objet. Si le scanner n'est pas face à l'objet et si la surface est irrégulière, la densité ne sera pas homogène et des masques ou zones d'ombre, vont inévitablement apparaître. Pour compenser ce problème et assurer la couverture la plus dense et la plus homogène possible, il faut multiplier les positions de numérisation autour de l'objet.

Si cette technique est devenue aujourd'hui très performante, le cas de la salle hypostyle de Karnak était particulièrement contraignant. En effet, les espaces entres les colonnes sont relativement étroits et empêchent d'avoir un recul suffisant pour capter convenablement les mesures. D'autre part, les 134 colonnes sont autant de " masques » qu'il faut contourner et qui par conséquent obligent à multiplier les stations de scannage. À ces contraintes s'est ajoutée l'obligation d'œuvrer sans gêner les circulations quotidiennes des très nombreux visiteurs.

La stratégie d'intervention fut donc longuement réfléchie afin d'optimiser le nombre de positions de l'appareil, tout en assurant une densité nécessaire et un bon recouvrement des mesures. Au 
total, ce sont près de 300 stations qui ont été installées. Un milliard de points ont été enregistrés, couvrant l'ensemble de la salle à raison d'un point tous les $5 \mathrm{~mm}$ en moyenne et faisant de cette opération l'un des plus gros chantiers de numérisation $3 \mathrm{D}$ de ces dernières années. Les points 3D peuvent être tellement denses que l'on parle de nuage de points.

Brut d'acquisition, ce nuage flotte dans l'espace. Pour qu'il soit exprimé dans un système connu de coordonnées (i.e. le système de coordonnées du site des Temples de Karnak), le scanner doit aussi numériser finement des repères disposés sur et autour de l'objet étudié et dont on aura préalablement déterminé les coordonnées par levé tachéométrique classique.

Ces données topométriques sont aujourd'hui traitées et ont permis de réaliser un modèle numérique $3 \mathrm{D}$ précis de chaque colonne.

Cette modélisation s'appuie sur la reconstitution fine de la surface de la colonne. Car aussi petit soit-il, l'espace entre chaque point mesuré par un scanner reste vide. La mesure est discrète et non continue. Les solutions informatiques développées pour cette technologie permettent de combler ce vide en reliant les points du nuage par des triangles. On parle de modèle maillé triangulé. Dans la mesure où la densité du nuage de points est suffisamment importante et homogène, le modèle maillé peut couvrir l'objet numérisé dans sa totalité. On dispose alors d'une archive 3D de l'objet, que certains qualifient même de moule numérique. De cette archive peut ensuite être extraire n'importe quelle information géométrique : il est par exemple possible de connaître la position et l'orientation exacte de l'axe de chaque colonne, de cartographier leur inclinaison, d'estimer finement le volume et donc le poids de chacune, de mesurer la surface des zones décorées.

\section{La campagne de relevé photogrammétrique}

Malgré la densité extrême des mesures qu'un scanner 3D est capable de fournir, l'information acquise reste exclusivement géométrique. Or, certaines géométries ne se distinguent pas par leur relief mais par leur couleur. C'est le cas de la majorité des décors pariétaux des colonnes. Ce problème est récurrent en archéologie et en architecture c'est pourquoi les scanners 3D sont souvent couplés à des chambres de prises de vue photogrammétriques calibrées. Par ailleurs, les capteurs photographiques ayant une résolution supérieure 
à celle des scanners, des données plus précises peuvent être enregistrées grâce à l'image. La calibration permet de lier géométriquement chaque pixel d'une photographie numérique prise depuis le scanner aux points 3D acquis dans le même temps.

Malheureusement, dans le cas de la salle hypostyle, les clichés photonumériques acquis directement depuis les scanners ne suffisent pas. En effet, l'incidence des rayons perspectifs des prises de vue, ne permet pas d'obtenir une résolution d'image homogène et de bonne qualité. Pour obtenir une couverture photographique exploitable, il convenait par conséquent d'optimiser la qualité des images grâce à des visées frontales tout en garantissant une résolution suffisamment fine pour enregistrer les détails.

Seule une campagne de prises de vues photogrammétriques stéréoscopiques réalisée dans les règles de l'art pouvait apporter une documentation nécessaire. Le cahier des charges était particulièrement exigeant et un compromis entre différents facteurs était nécessaire : résolution des images inférieure à $3 \mathrm{~mm}$, précision du calage de ces images dans le même système que les données laser, dimension de la salle et temps d'acquisition raisonnable. Comment alors réaliser les prises de vues en respectant les contraintes de la photogrammétrie?

L'acquisition photographique a été entreprise lors d'une seconde mission, par une équipe de l'École nationale Supérieure de Géographie (ENSG-IGN); à la tête de celle-ci, Yves Egels, Daniel Schelstraete et Laure Chandelier ont coordonné huit étudiants ${ }^{23}$. En fonction de la géométrie de la salle et du choix des appareils photographiques une configuration théorique optimale a été déterminée puis vérifiée par des simulations informatiques.

Ces différents tests ont permis de définir une procédure efficace pour photographier près d'un demi-hectare de décors avec une définition suffisante. Entre 32 et 40 clichés selon les deux types de colonnes, ont été nécessaires afin de parvenir à une définition de deux millimètres par pixel. Plus de 4400 images ont été enregistrées par

23. L'équipe était composée des élèves géomètres en $2^{\text {ème }}$ année de l'ENSG : Sylvain Audel, Julie Cheikbossian, Stéphane Coetmeur, Basile Jouan, Jeremy Maillard, Cédric Melet, Nicolas Pourre, David Bourdon ; Yvan Robin (Atm3d), des topographes du Cfeetk : Antoine Marmousez et Louis Elia; des photographes du Cfeetk : Jean-François Gout, Clément Apffel et Lucie Moraillon ; de Gaël Pollin (photographe/CEAlexandrie) et Laurent Borel (architecte/CNRS/CEAlexandrie), ainsi que du Raïs Mahmoud Farouk, Hamade abdu Khedaoui, Ala Ibrahim, Mohamed Abdou et Sayed. Les travaux se sont déroulés du 26 avril au 25 mai 2008. Un compte rendu des travaux est consultable sur le site de 1'ENSG : http://www.ensg.eu/Leve-des-colonnes-de-la-salle-hypostyledu-temple-de-Karnak-Egypte. 


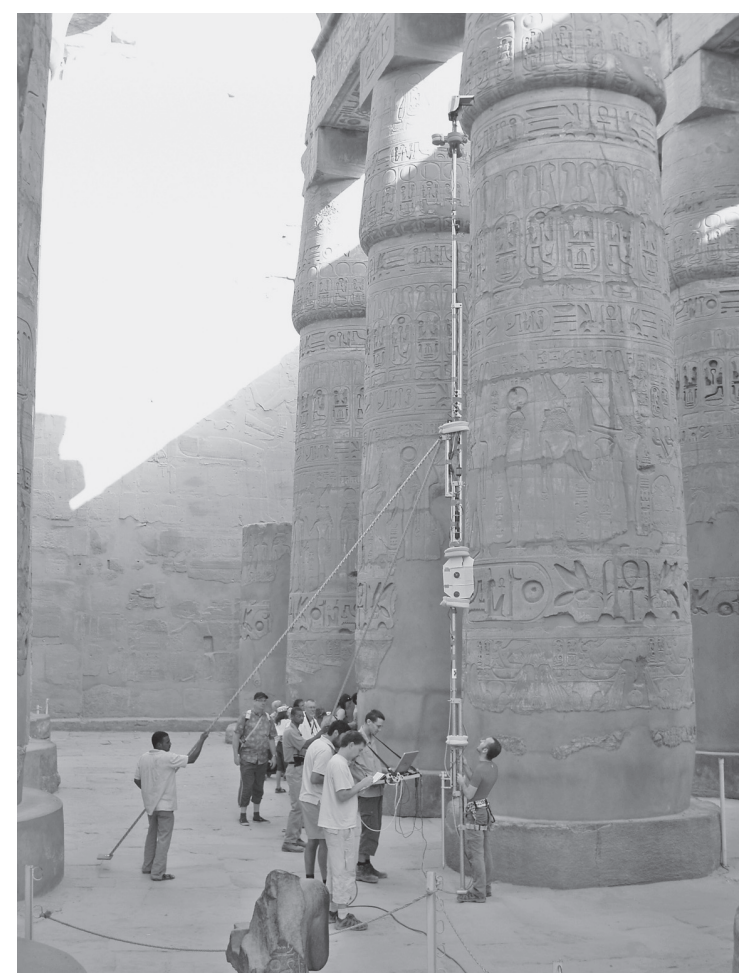

FIG. 2. - La « perche » mise au point par l'équipe de l'ENSG. Les quatre appareils photographiques calibrés qui y son fixés, sont déclenchés simultanément à partir d'un ordinateur (E. Laroze).

tranches verticales à l'aide d'une perche de huit mètres sur laquelle quatre appareils photographiques étaient fixés à des hauteurs différentes (fig. 2). Un ordinateur, installé à la base du mât permettait de contrôler le cadrage et d'assurer le déclenchement simultané des quatre appareils. En raison du manque de recul suffisant, les décors des colonnes périphériques orientés vers les murs ont été photographiés « manuellement », depuis une échelle de huit mètres (fig. 3).

Trois semaines ont été nécessaires pour acquérir l'ensemble des clichés ; un temps record, compte tenu du nombre de photographies mais surtout des contraintes d'éclairage, très changeant au cours de la journée. En effet, en plein jour, la lumière créée des ombres portées et des variations d'intensité importantes sur les colonnes, ce qui a astreint les photographes à se déplacer de façon aléatoire 


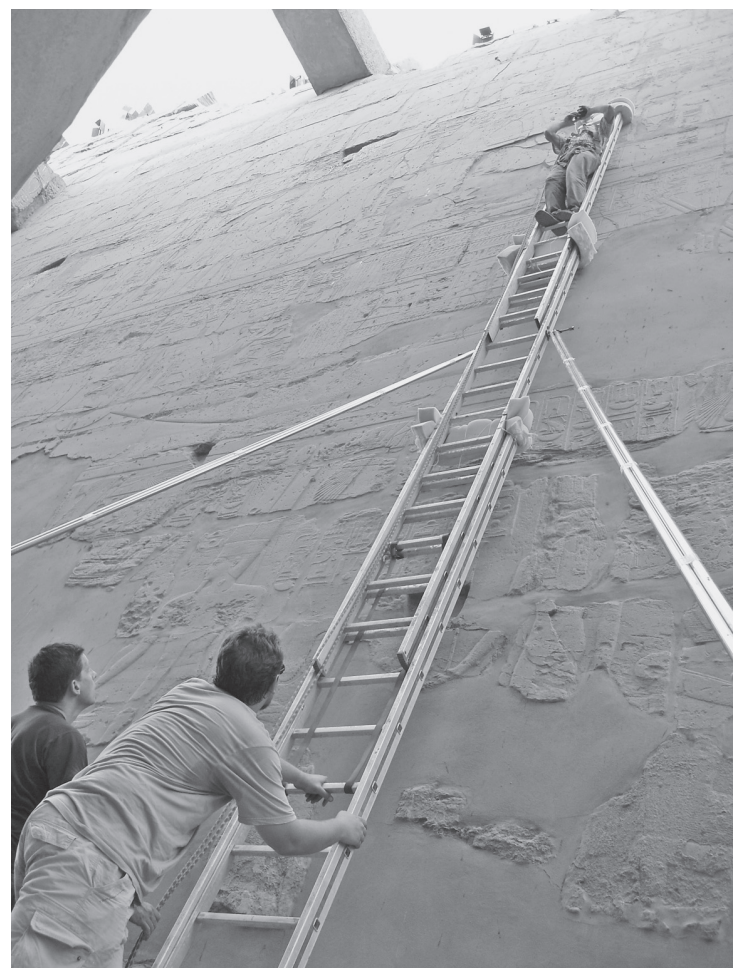

FIG. 3. - Le long des parois, les prises de vue ont été réalisées « manuellement » depuis une échelle (E. Laroze).

dans la salle, pour contribuer à homogénéiser la radiométrique des photographies. Malgré ces mesures, ces problèmes persisteront mais seront partiellement corrigés par les outils informatiques.

Cette deuxième étape clôt la phase d'enregistrement des données sur le terrain. Elle précède une nouvelle phase : le traitement des images photogrammétriques et leur association aux modèles 3D, dont l'objectif final est de produire un déroulé photographique haute résolution de chaque colonne.

Dans cette ultime phase, qui est en cours, il s'agit de réaliser une aérotriangulation des prises de vues, à savoir la détermination millimétrique du géoréférencement en position et orientation des images dans le système du modèle 3D laser. Cette étape est déterminante puisqu'elle permet d'associer n'importe quel point du modèle 3D d'une colonne à son image dans les prises de vues qui la couvre. 


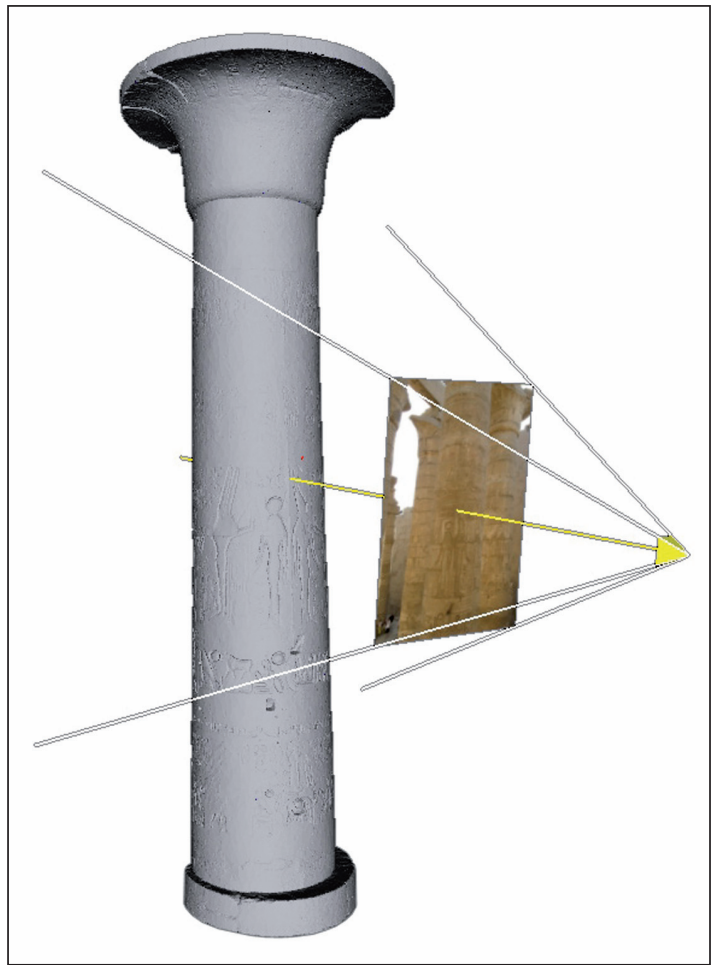

FIG. 4. - Principe de projection d'une image photogrammétrique sur le modèle 3D (B. Chazaly).

La représentation graphique globale, complète et détaillée des décors des colonnes s'articule autour de cette association, modélisée mathématiquement par les équations photogrammétriques. Pour chaque photographie, elle permet de corriger les déformations dues à la perspective, les imperfections de l'objectif utilisé, le relief de l'objet lui-même et sa forme cylindrique. Enfin, le processus final, qui consiste à projeter une image sur un support (fig. 4 et 5), exploite les mêmes transformations mathématiques qui sont utilisées pour générer un planisphère. Dans notre cas, la projection est cylindrique. Chaque pixel des photographies est replacé à son emplacement exact dans un système de coordonnées cylindrique. Cette opération permet de développer numériquement la surface de chaque colonne de manière à la mettre à plat, en 2D (fig. 6). On obtient alors un document technique offrant aux épigraphistes une représentation cohérente et détaillée de l'ensemble des décors. 


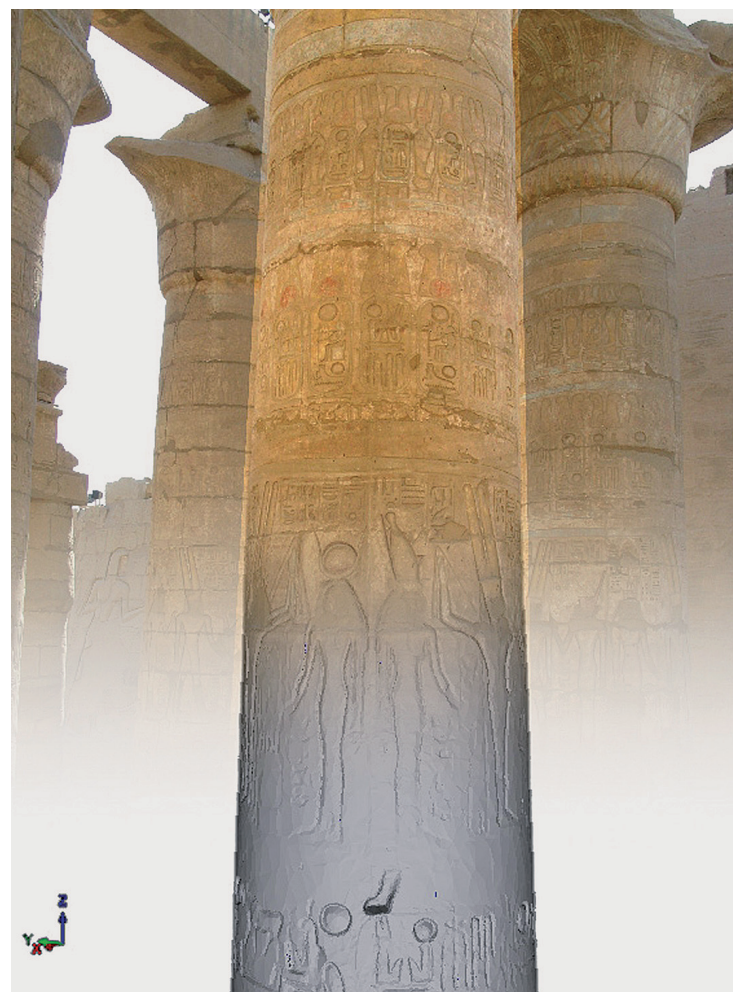

FIG. 5. - Superposition d'un cliché photographique calibré avec une vue du modèle 3D. Grâce aux informations photogrammétriques il est possible de retrouver le positionnement exact de l'appareil photographique et par conséquent de faire correspondre un cliché avec un modèle numérique (B. Chazaly).

\section{Conclusions}

Les données seront traitées cette année. Un catalogue présentant un déroulé ortho-photographique des décors de chaque colonne et une cartographie complète de la salle seront réalisés (fig. 7). Dans la perspective d'une publication, cette documentation inédite sera confiée au docteur Peter Brand de l'Institute of Egyptian Art and Archaology, de l'Université de Memphis et au professeur d'histoire Jean Revez de l'Université de Québec à Montréal, qui mènent depuis plusieurs années l'étude épigraphique de la salle ${ }^{24}$.

24. http://history.memphis.edu/hypostyle. 


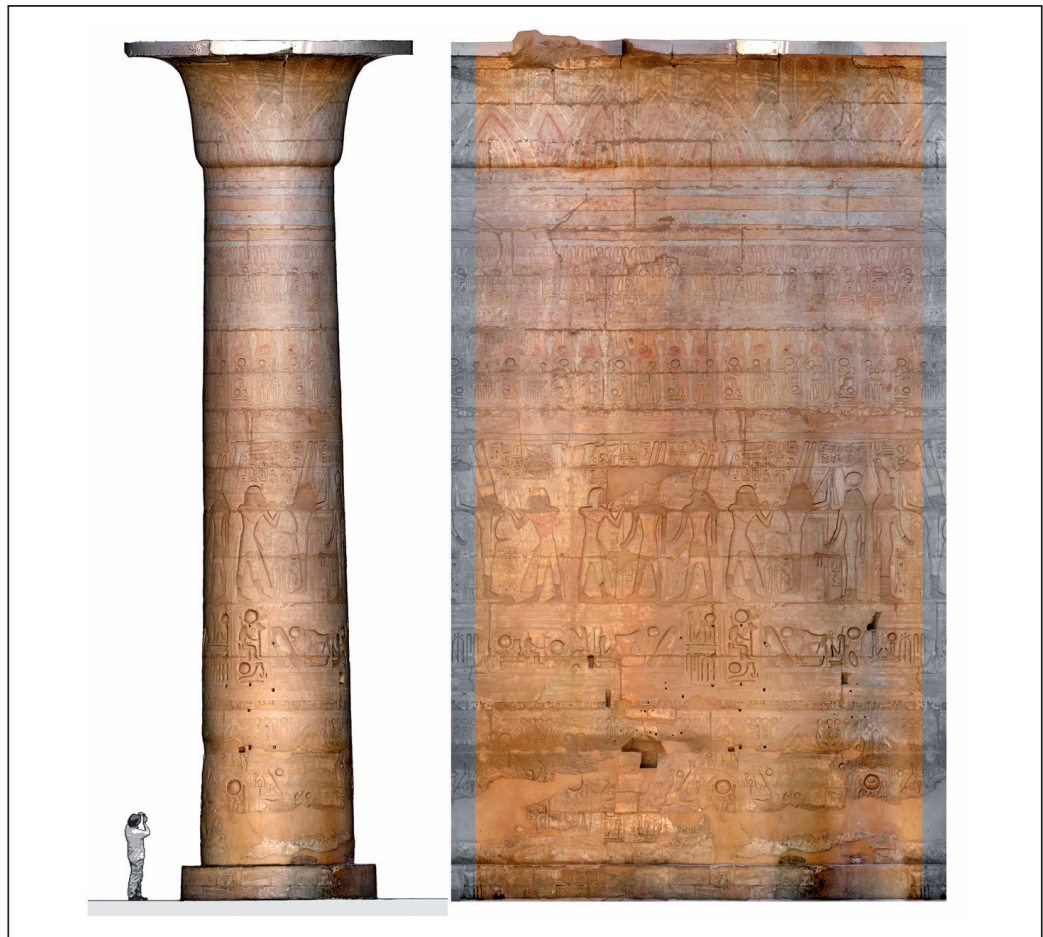

FIG. 6. - Modèle numérique coloré de la colonne $2 \mathrm{n}$ et son développé orthophotographique correspondant (Atm3d/Cfeetk).

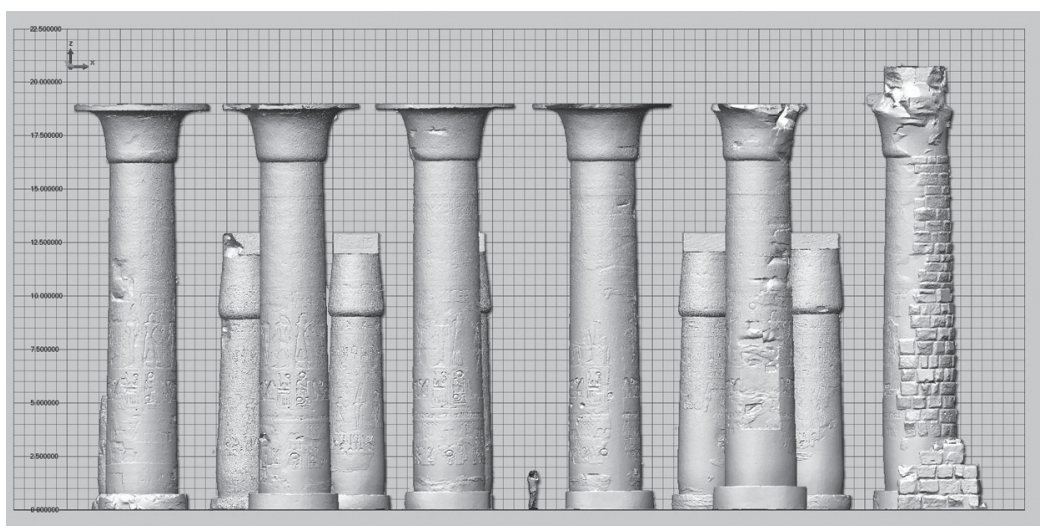

FIG. 7. - Vue géométrale du modèle 3D de la partie nord de la salle hypostyle (Atm3d/Cfeetk). 
Enfin, à partir des documents numériques il sera possible d'associer ou de dissocier une image photographique d'un modèle 3D, c'est-àdire d'enrouler chaque image haute résolution pour la draper sur le maillage 3D de la colonne correspondante. Le modèle texturé aura acquis une très haute plus-value graphique puisqu'il rendra la navigation virtuelle dans la salle hypostyle envisageable. C'est pourquoi, il est aussi projeté de diffuser les résultats de ce programme à un public plus large, sous la forme d'une interface interactive (DVD, site internet...) afin que chacun puisse mieux interpréter ou plus simplement contempler, sous un œil nouveau, cette admirable œuvre architecturale.

MM. Nicolas GRIMAL et Robert MARTIN interviennent après cette note d'information. 
